\title{
LA GASTRONOMÍA EN EL MARKETING TURÍSTICO
}

\author{
Francesc Fusté-Forné*
}

Resumen

El objetivo de este artículo es analizar el uso de la gastronomía en el marketing turístico. La gastronomía es un factor cada vez más importante dentro del abanico de recursos turísticos de cualquier destino. El turismo gastronómico tiene un interés para todos los actores: gestores de turismo, intermediarios, productores y visitantes. Por un lado, esto se refleja en el gasto turístico vinculado al consumo gastronómico, pero también en la motivación de experimentar los productos auténticos, las recetas locales 0 eventos y museos centrados en la identidad culinaria. El interés hacia la gastronomía se traslada a la promoción de los destinos turísticos, que se convierten también en destinos gastronómicos. Para ilustrarlo, la investigación se sirve de ejemplos de promoción turística de la gastronomía de los países de América Latina. El método utilizado es el estudio de caso descriptivo. Los resultados demuestran hasta qué punto en la actualidad se ha incluido la gastronomía como un factor de atracción turística en el contexto de la promoción de los destinos.

Palabras Clave: Autenticidad. Comunicación Cultural. Gastronomía. Marketing. Promoción. Turismo Gastronómico.

\section{GASTRONOMIA EM MARKETING DE TURISMO}

\section{Resumo}

Este trabalho pretende estudar o uso da gastronomia no marketing turístico. A gastronomia é um fator crucial no que se refere à atração turística. 0 turismo alimentar é, portanto, um campo fundamental para as partes interessadas do turismo: gerentes, agências de viagens e operadores turísticos, produtores e visitantes. Esta importância é derivada da despesa turística no consumo da gastronomia, mas também da forte motivação em relação à experiência de produtos autênticos, receitas locais ou eventos e museus com base em identidades culinárias. 0 interesse pela gastronomia também atinge a promoção de destinos. Para ilustrar isso, a pesquisa analisa exemplos de promoção do turismo gastronômico na América Latina. O método utilizado é o estudo de caso descritivo. Os resultados mostram até que ponto a gastronomia está incluída nas práticas de turismo no contexto da promoção de destinos.

Palavras Chave: Autenticidade. Comunicação cultural. Gastronomia. Marketing. Promoção. Turismo gastronômico.

\section{GASTRONOMY IN TOURISM MARKETING}

This paper aims to study the use of gastronomy in tourism marketing. Gastronomy is a crucial factor with regards to tourism attraction. Food tourism is therefore a key field for tourism stakeholders: managers, travel agencies and tour operators, producers and visitors. This importance is derived from the tourist expenditure on gastronomy consumption, but also from the strong motivation regarding the experience of authentic produce, local recipes, or events and museums based on culinary identities. The interest on gastronomy also reaches the promotion of destinations. To illustrate it, the research analyses examples of tourism promotion with mregards to gastronomy in Latin America. The method used is based on a descriptive study case. Results show to what extent gastronomy is included within tourism practices in the context of destination marketing.

Keywords: Authenticity. Cultural communication. Gastronomy. Marketing. Promotion. Food tourism.

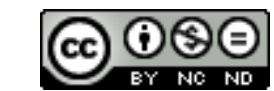

Licenciada por Creative Commons Atribuição Não Comercial / Sem Derivações/ 4.0 / Internacional

\footnotetext{
* Doutor em Turismo, Direito e Negócios pela Universidade de Girona/Espanha. Profesor Asociado, Facultad de Turismo, Universitat de Girona/Espanha. Pesquisador visitante na Lincoln University/Nova Zelândia. Sua pesquisa centra-se no turismo rural e turismo alimentar, e suas contribuições para o desenvolvimento local. Interessa-se também pelo estudo da interação entre experiências e turismo tanto no indústria e paisagem. [ researchexperiencetourism@gmail.com ]
} 


\section{INTRODUCCIÓN}

La gastronomía es un factor de atracción clave en el contexto de la oferta turística de los destinos. El turismo gastronómico tiene un interés cada vez mayor tanto para gestores e intermediarios, como para los productores y los visitantes. Al mismo tiempo, tal y como afirman Chen y Huang (2016), la creciente importancia de la popularidad del turismo gastronómico ha implicado también un volumen de producción científica alrededor de esta temática sin precedentes. Dicha literatura sobre el turismo gastronómico se ha desarrollado desde varios puntos de vista y se puede encontrar en las principales revistas científicas de impacto en el ámbito, tanto en inglés como en otros idiomas tales como el español, portugués, francés o chino, entre otros. Este interés de los distintos actores hacia la gastronomía se debe principalmente al hecho que los visitantes tienen una creciente motivación hacia el descubrimiento de las culturas locales a través de su comida, por ejemplo, probando un plato tradicional 0 una bebida típica (HJALAGER; CORIGLIANO, 2000).

El turismo gastronómico es una de las especializaciones turísticas con mayor relevancia, tanto como motivación principal como en relación al consumo inherente a cualquier desplazamiento turístico. Las marcas turísticas, tanto de ciudades como de regiones y países, tienden a presentar una oferta de productos gastronómicos cada vez mayor y más heterogénea. Como consecuencia, la importancia de la comida en la experiencia turística gastronómica se refleja en el consumo turístico. Evidentemente, este interés gastronómico se traslada a la promoción de los destinos turísticos (BARROCO; AUGUSTO, 2016). Poco a poco, todas las regiones se convierten en cierta forma en destinos gastronómicos, donde la cocina y la gastronomía desarrollan un rol crucial.

El objetivo de este artículo es ver si efectivamente se desarrolla el marketing gastronómico de forma generalizada $y$ en consecuencia reflexionar sobre el uso de la gastronomía en el contexto del marketing turístico. El hecho de analizar el papel de la gastronomía en el contexto de la promoción de los destinos es relevante para poder conocer sobre qué elementos se sustenta la oferta turística gastronómica de estos destinos, y en consecuencia poder adaptarla con el objetivo de mejorar la gestión de la oferta del destino y en particular beneficiar el desarrollo regional a partir de las prácticas del turismo gastronómico.

Tal y como afirma Cabrera (2013), la gastronomía engloba un sector que es cada vez más amplio y competitivo, lo cual hace necesario el desarrollo de estrategias de marketing que puedan beneficiarse del atractivo turístico de la gastronomía. En este sentido, el turismo gastronómico es un ingrediente clave del marketing de destinos (COHEN; AVIELI, 2004; HALL; SHARPLES, 2003; HJALAGER; RICHARDS, 2002), ya que implica una elevada dosis de diferenciación. De esta forma, la asociación de la gastronomía con las identidades regionales y nacionales ofrece una oportunidad a los destinos para utilizar la gastronomía y los alimentos y platos locales en sus estrategias de comunicación y comercialización (OKUMUS; OKUMUS; MCKERCHER, 2007). Hall (2003) también afirma que todos los recursos relacionados con la cocina y la gastronomía representan un elemento de promoción turística clave para la competitividad del marketing de destinos. Por su lado, Du Rand y Heath (2006) destacan que una de las líneas de investigación en el contexto del estudio del turismo gastronómico debe centrarse en cómo los alimentos y la gastronomía pueden contribuir a las estrategias de comercialización del turismo, dada su especial relevancia como atractivo de la oferta.

En la misma dirección que la investigación de Chaves y Costa (2014), el tema que vertebra este artículo es el turismo gastronómico como fenómeno de consumo turístico, considerando el papel fundamental que la gastronomía puede desempeñar en la comercialización de los destinos turísticos. Así pues, para llevar a cabo el estudio, se plantean las siguientes preguntas de investigación:

1- ¿La gastronomía se incluye en la actualidad de forma generalizada en la promoción de los destinos turísticos?

2- ¿Por qué la gastronomía se utiliza de forma tan recurrente en el marketing turístico?

La investigación, basada en el método del estudio de caso descriptivo, se sirve de los productos, experiencias y regiones gastronómicas que conforman la promoción turística de la gastronomía de los países de América Latina, en particular los de América del Sur. En concreto, se analizan las diferentes propuestas gastronómicas que los organismos nacionales de gestión y promoción turística de estos países promocionan como parte de su oferta turística. Los resultados permiten observar cómo los países incluyen el componente gastronómico en su promoción turística y pueden ser útiles para la gestión y proyección de nuevas oportunidades empresariales entorno el turismo gastronómico.

La estructura del texto, después de la introducción, parte de una sección teórica que aborda 
la relación entre el turismo y la gastronomía. En este sentido, se presenta la gastronomía como diferencial en la identidad $y$ en el marketing de destinos turísticos. A continuación se incluye la sección que analiza la gastronomía en la promoción turística a partir de los resultados obtenidos de los países de América del Sur, que son, por orden alfabético, los siguientes: Argentina, Bolivia, Brasil, Chile, Colombia, Ecuador, Paraguay, Perú, Uruguay y Venezuela. Finalmente, se pueden observar las conclusiones del estudio, limitaciones y otras líneas de investigación, así como las referencias bibliográficas utilizadas.

\section{MÉTODO DE ESTUDIO}

El método utilizado para la elaboración de este artículo se basa en el estudio de caso descriptivo. En particular, de acuerdo con Yin (2014), la metodología del caso de estudio es adecuada cuando se exploran preguntas de investigación que pretenden responder al cómo y al por qué. Así pues, este estudio analiza cómo la gastronomía se incluye en la promoción de los destinos turísticos, y, en consecuencia, pretende discutir los motivos por qué la gastronomía es un recurso tan habitual en las estrategias de marketing turístico en la actualidad. Para ello, se recopilan los elementos que se utilizan para promocionar el turismo gastronómico por parte de los organismos oficiales de turismo de los países de América del Sur, tal y como se mencionaba en la sección anterior. En este sentido, la recogida de datos se ha llevado a cabo a partir del estudio de fuentes secundarias, $y$, en particular, de los portales web de estos organismos, incluyendo cualquier ejemplo que haga referencia a la práctica del turismo gastronómico.

\section{TURISMO Y GASTRONOMÍA}

La gastronomía es parte de la cultura de los territorios, y uno de los componentes más importantes del sentido de pertenencia a un lugar y su autenticidad. La Organización Mundial del Turismo (2012) destaca la gastronomía como parte de la experiencia turística, a través de la cual los visitantes y turistas pueden conocer el patrimonio de un destino. En esta dirección, muchos autores reconocen los beneficios de la conexión entre turismo y gastronomía para con el desarrollo regional (HALL; SHARPLES; MITCHELL; MACIONIS; CAMBOURNE, 2003; HJALAGER; RICHARDS, 2002; SIDALI; KASTENHOLZ; BIANCHI, 2015; SIMS, 2009). Esto es debido a la estrecha relación del turismo gastronómico con los productos locales, las actividades del sector primario, y el paisaje no solo cultural sino también natural (BERNO; LAURIN; MALTEZAKIS, 2014; FUSTÉ-FORNÉ, 2016b). Estos elementos representan la base para el posterior desarrollo del turismo gastronómico.

A la vez, la puesta en valor de la gastronomía a través del turismo es una forma de especialización, diferenciación y diversificación de la oferta turística, lo cual ayuda a reducir los efectos negativos de la estacionalidad de la demanda en muchos destinos. La gastronomía se puede visitar durante cualquier momento del día, bajo cualquier condición meteorológica y por lo tanto indistintamente durante todo el año (KIVELA; CROTTS, 2006). Esto la convierte en un recurso fundamental entorno el cual los gestores turísticos tienen un interés estratégico creciente. Además, estudios recientes confirman que la atracción de la gastronomía es una motivación cada vez más importante para los visitantes y turistas (HALL, 2016), con una demanda creciente también vinculada a productos gastronómicos con certificados de calidad (BIGNÉ, 2011; TRESSERRAS; MEDINA, 2008). Esta motivación por la gastronomía se produce con el objetivo de consumir productos locales, platos tradicionales, museos 0 eventos gastronómicos, recorrer itinerarios gastronómicos y visitar centros de producción y distribución.

A lo largo de los últimos años, tanto los académicos como los gestores y promotores turísticos han puesto el punto de mira en la gastronomía. ¿Pero, qué elementos se promocionan para dar a conocer la identidad gastronómica de los destinos? Aunque no hay estudios en esta dirección, una de las principales razones de promoción de la gastronomía es el hecho que la particularidad que ofrece la producción localizada en un determinado lugar genera una atracción especial hacia él, ya que se enmarca en una identidad paisajística única (LEE; WALL; KOVACS, 2015), que a la vez ayuda a hacer frente al fenómeno de la globalización. Tal y como afirman Bèssiere (2001) o Kim y Eves (2012), cuando se consumen especialidades gastronómicas en su lugar de origen se consigue una apropiación efímera y simbólica de la cultura, la historia, el paisaje, o el clima de una región. No hay duda que la comida y la gastronomía son un factor clave del atractivo de los destinos (BJÖRK; KAUPPINEN-RÄISÄNEN, 2016; CROCE; PERRI, 2011; SIMS, 2009).

\subsection{La gastronomía como diferencial en la identidad y en el marketing de destinos turísticos}

Además de las obras de Kotler (ver por ejemplo KOTLER ET AL, 2011), los estudios de Bigné (1996; 2005) son de los que más ampliamente han 
desarrollado la conexión entre turismo y marketing. Así mismo, aunque los trabajos entorno al marketing gastronómico son escasos, son varios los autores que han destacado la gastronomía como uno de los ingredientes más importantes cuando se comunica la identidad de un destino (CROCE; PERRI, 2011; DU RAND; HEATH; ALBERTS, 2003; OKUMUS; OKUMUS; MCKERCHER, 2007).

En concreto, la promoción de la gastronomía es una manifestación de las narrativas patrimoniales y nacionalistas de un lugar (EVERETT, 2016). Por lo tanto la gastronomía es un atributo crucial en la formación de la imagen del destino (BIGNÉ, 2011; ROYO, 2011). Esto tiene especial relevancia en referencia a productos con etiquetas de calidad como las denominaciones de origen, debido a su vinculación con el territorio de origen, la producción ecológica, la estrecha relación con el medio ambiente y su vinculación con la naturaleza y el entorno rural (CALVO, 2011). Además, la gastronomía sirve como factor de diferenciación, y los turistas la perciben como una experiencia de ocio y acercamiento cultural, que es la suma de las idiosincrasias de los paisajes históricos, socioculturales y naturales de un destino (FUSTÉ-FORNÉ, 2015; HILLEL; BELHASSEN; SHANI, 2013; SIMS, 2009), tal y como se apuntaba anteriormente.

La importancia de la gastronomía en el contexto de la práctica turística es tan evidente como la necesidad que todos los turistas tienen de comer durante sus viajes (RICHARDS, 2002), siendo este, en consecuencia, uno de los principales gastos de la demanda turística, y por lo tanto parte estructural de la oferta de cualquier destino. En este sentido, el presupuesto de los turistas para con el consumo alimentario y gastronómico no incluye únicamente la compra de productos alimentarios locales, sino que se refiere a un conjunto más amplio de productos y experiencias turísticas, que se adquieren también a modo de souvenirs (FUSTÉ-FORNÉ, 2016a; HALL; SHARPLES, 2003; HENDERSON, 2009). La participación en la cultura gastronómica local forma parte de una experiencia turística auténtica que tiene como objetivo la búsqueda de las tradiciones culinarias del destino que se está visitando, así como el mayor conocimiento del estilo de vida de sus habitantes, lo cual se consigue a través de la gastronomía. Tal y como lo reflejan Gyimóthy y Mykletun (2009), la gastronomía no deja de ser una forma de expresión artística, donde los productos y las experiencias culinarias son manifestaciones de la identidad local, y reflejan los sentidos de lugar de cada región. Esto se debe a la pertenencia de la gastronomía a la cultura (FERRERO, 2002), y a la conexión de ambas con el territorio (LONG, 2010), lo cual representa una oportunidad para su promoción turística.

En la relación entre marketing y turismo, ambas son disciplinas que en la práctica se han desarrollado de forma muy paralela a lo largo de los últimos dos siglos. Las tipologías turísticas han ido evolucionando, así como las formas de promoción de los destinos. Así pues, tal y como afirma Weill (2003), las empresas ferroviarias de la primera mitad del siglo XIX ejemplificaron esta utilización inicial de la promoción vinculada al desplazamiento, también turístico. Los trenes se sumaron rápidamente a la actividad turística tal y como lo demuestran los desplazamientos a determinados eventos 0 los mismos trenes turísticos que llegan hasta nuestros días como un producto, en muchos casos, de lujo. En este sentido, la publicidad en prensa o los carteles relacionados con el transporte ferroviario se consideran un primer embrión de lo que más tarde se ha estudiado como marketing turístico.

Por ejemplo, en el caso español, algunos autores afirman que los orígenes de la promoción turística son relativamente recientes (HERNÁNDEZ, 2008). Según Hernández (2008), los primeros anuncios públicos que promocionaron destinos turísticos españoles se remontan a finales de la década de los años 20 del siglo pasado, y sucedieron en el contexto de las políticas de fomento del sector turístico del Patronato Nacional de Turismo, creado en el año 1928. No obstante, cabe destacar también otros organismos anteriores como por ejemplo la Sociedad de Atracción de Forasteros, creada en el año 1908 con el objetivo de promocionar la actividad turística en Catalunya (BLASCO, 2009).

La importancia del marketing y la publicidad en el mundo actual es transversal, y aun mas con el desarrollo de Internet y la influencia de las redes sociales. Sobre todo a partir de la finalización de la Segunda Guerra Mundial, de acuerdo con Hernández (2008), fue cuando empezaron a crecer con fuerza las inversiones en publicidad, ya que las empresas se van dando cuenta de su rol, que va más allá de un gasto asociado a una estrategia de comercialización, y se asocia a una inversión vinculada a la creación de una imagen de marca. Esto es especialmente relevante en el sector turístico, donde el marketing permite a los visitantes y turistas conocer el producto con anterioridad - es decir, materializar el servicio turístico que en esencia se caracteriza por su intangibilidad, inseparabilidad 0 carácter perecedero (ver KOTLER ET AL, 2011) - .

El marketing de los destinos turísticos es un claro ejemplo de competitividad de los mismos (CROUCH; RITCHIE, 1999; RITCHIE; CROUCH, 2000). Ahora bien, se trata de un ámbito de estudio 
complejo debido a la intervención de una gran diversidad de actores (FRUSTIER, 2009), tanto públicos como privados, y en un contexto donde las tendencias turísticas actuales de especialización de la oferta, implican una constante necesidad de adaptarse a los deseos cambiantes de la demanda. Esto aun adquiere más importancia considerando la elevada competencia entre destinos. Por lo tanto, la provisión de una información amplia y detallada de los recursos permite a los turistas desarrollar un conocimiento del destino lo más amplio posible, donde es necesario implementar las estrategias de marketing no solo desde el punto de vista tradicional del marketing de destinos - incrementar el número de visitantes (BIGNÉ; FONT; ANDREU, 2000) -, sino también centrar la estrategia de los planes de marketing en el desarrollo regional, la responsabilidad social y la sostenibilidad.

Desde el punto de vista de la gestión del marketing turístico y gastronómico, los alimentos representan uno de los elementos más importantes de la cartera de productos de un destino; así, a través de su consumo, los visitantes y turistas adquieren una experiencia cultural auténtica, también en relación a las poblaciones inmigrantes de un determinado lugar (OKUMUS; OKUMUS; MCKERCHER, 2007). Por lo tanto, y tal y como se apuntaba en la sección introductoria, la gastronomía debe desarrollar un papel clave en la comercialización de destinos, tanto a nivel local y regional, como nacional. En esta dirección, autores como Bessière (1998) afirman que la promoción y el marketing sobre gastronomía son el reflejo de la identidad social y cultural, así como la comunicación y el intercambio de valores vinculados a la alimentación y la cocina.

En definitiva, poniendo el punto de mira en la importancia de la gastronomía, algunos estudios destacan el papel determinante que la comida juega en las estrategias de marketing de los destinos y en particular en el marketing gastronómico (CABRERA, 2013), y en algunos casos emergiendo como la principal herramienta de promoción (DU RAND; HEATH, 2006). De forma más específica, otros estudios han analizado por ejemplo el rol de los festivales gastronómicos para la promoción de los destinos (LEE; ARCODIA, 2011). Aun así, en general, los mismos autores reconocen que se trata de un terreno que en determinados destinos puede desarrollarse más ampliamente y de forma coordinada entre los diferentes actores. Esto permitiría avanzar en la consolidación de una estrategia coherente para con el turismo gastronómico.

\subsection{La gastronomía en la promoción turística}

Para comprobar si efectivamente la gastronomía se ha incluido de forma generalizada en la promoción de los destinos turísticos, se han analizado los casos de promoción turística de los países de América Latina, concretamente los ubicados en América del Sur. Con el fin de dar respuesta a las preguntas de investigación definidas al principio del artículo, el estudio se ha llevado a cabo a partir de fuentes secundarias, en concreto analizando los contenidos gastronómicos sobre gastronomía de los sitios web de los organismos oficiales de gestión y promoción del turismo de cada país.

En la tabla 1 se puede observar la relación de países y sus organismos de gestión y promoción turística. En la tercera columna de la tabla se enumeran los principales recursos, experiencias y regiones gastronómicas que dichos organismos resaltan como los más destacados de cada país. En concreto, estos 95 ejemplos se pueden clasificar, de acuerdo con las diferentes prácticas de turismo gastronómico, en productos culinarios - por ejemplo, el cacao -, experiencias gastronómicas - por ejemplo, comer tortas fritas cuando llueve -, y regiones - por ejemplo, La Rioja -. Como se observa en el análisis más abajo, cada país da visibilidad a su gastronomía de una forma distinta.

Es importante mencionar que la relación de los elementos que reflejan la identidad gastronómica no es exhaustiva y únicamente es una muestra exploratoria obtenida a partir de las instituciones analizadas. De esta manera, la lista de los recursos gastronómicos que tienen la capacidad de mostrar la autenticidad de los países es mucho más amplia y responde también a particularidades regionales que no se reflejan en este artículo. Esto se debe a que el análisis se hace únicamente desde una perspectiva nacional. 
Tabla 1. Los productos gastronómicos de los países de América del Sur según su enumeración en los sitios web de los organismos de gestión y promoción turística estatales.

\begin{tabular}{|c|c|c|}
\hline País & $\begin{array}{l}\text { Organismo consultado en relación a } \\
\text { la gestión y la promoción turística }\end{array}$ & Ejemplos de productos, experiencias y regiones gastronómicas \\
\hline Argentina & $\begin{array}{llll}\text { Instituto Nacional de } & \text { Promoción } \\
\text { Turística } & & & \\
\end{array}$ & $\begin{array}{l}\text { La nueva cocina porteña } \\
\text { Sabores del mar y meriendas con historia en Chubut } \\
\text { Sabores del fin del mundo } \\
\text { La Rioja, viñedos entre olivares } \\
\text { San Juan, tierra del Syrah } \\
\text { Chivitos: el sabor de San Luis } \\
\text { Sabores de Tucumán } \\
\text { Los vinos del valle patagónico } \\
\text { El cordero, delicia patagónica } \\
\text { Catamarca, vinos en la Ruta del Adobe } \\
\text { Sabor a tradición -e innovación- en las cocinas de Buenos Aires } \\
\text { Sabores de la chacarera } \\
\text { Salta: la Ruta del Vino más alta del mundo } \\
\text { Neuquén, vinos de la Patagonia en el camino de los dinosaurios } \\
\text { Chocolates, cervezas artesanales y ahumados en la Patagonia } \\
\text { andina } \\
\text { Sabores rosarinos, con identidad propia } \\
\text { Sabores serranos } \\
\text { Córdoba, artesanía en vinos } \\
\text { Mendoza, capital mundial del Malbec } \\
\text { Cocina de autor en la cuna del Malbec } \\
\text { En el sur de Mendoza, viñedos entre los ríos } \\
\text { Mendoza Este, viñedos con historia } \\
\text { Valle de Uco, innovación en vinos } \\
\text { Sabores del Litoral } \\
\text { Sabores ancestrales en la Quebrada de Humahuaca } \\
\text { Sabores del Chaco }\end{array}$ \\
\hline Bolivia & $\begin{array}{l}\text { Viceministerio de Turismo / Ministerio } \\
\text { de Culturas y Turismo / Empresa } \\
\text { Estatal Boliviana de Turismo (Boltur) }\end{array}$ & Tarija fiesta de sabores con altura \\
\hline Brasil & $\begin{array}{llll}\text { Empresa } & \text { Brasileña } & \text { de } & \text { Turismo } \\
\text { (Embratur) } & & & \\
\end{array}$ & $\begin{array}{l}\text { Las maravillas de Bahía } \\
\text { Los innumerables sabores del Mercadão }\end{array}$ \\
\hline Chile & $\begin{array}{l}\text { Ministerio de Economía, Fomento y } \\
\text { Turismo }\end{array}$ & $\begin{array}{l}\text { Gastronomía del Mundo } \\
\text { Gastronomía Típica } \\
\text { Enoturismo }\end{array}$ \\
\hline Colombia & $\begin{array}{l}\text { Ministerio de Comercio, Industria y } \\
\text { Turismo }\end{array}$ & $\begin{array}{l}\text { Descubre el sabor de Soledad } \\
\text { Diviértete en el Festival del Arroz } \\
\text { Rincón Payanés } \\
\text { El Cuy } \\
\text { Come hormiga culona } \\
\text { Prueba las achiras de Altamira } \\
\text { Conoce la piña de Lebrija } \\
\text { Prueba el sancocho en Ginebra } \\
\text { Disfruta de un municipio dulce } \\
\text { Prueba el chorizo Santarrosano } \\
\text { Congreso Nacional Gastronómico de Popayán } \\
\text { Visita Luruaco } \\
\text { Prueba la longaniza de Sutamarchán } \\
\text { La posta negra cartagenera } \\
\text { Disfruta del sabor de El Jardín } \\
\text { Disfruta de los platos de Chía } \\
\text { Tour Gastronómico } \\
\text { Disfruta del pan de leña en Tabio } \\
\text { Recorre la zona G }\end{array}$ \\
\hline Ecuador & Ministerio de Turismo & Costa del Pacífico \\
\hline
\end{tabular}




\begin{tabular}{|c|c|c|}
\hline & & $\begin{array}{l}\text { Sierra } \\
\text { Amazonía } \\
\text { Galápagos } \\
\text { Cacao } \\
\text { Café } \\
\text { Granos y cereales } \\
\text { Frutas } \\
\text { Pescados y mariscos } \\
\text { Tubérculos } \\
\text { Festival del Queso } \\
\text { Fanesca } \\
\text { Colada morada } \\
\text { Torta negra de Jueves Santo } \\
\end{array}$ \\
\hline Paraguay & $\begin{array}{l}\text { Secretaría Nacional de Turismo - } \\
\text { SENATUR }\end{array}$ & $\begin{array}{l}\text { El Tereré } \\
\text { Comidas Típicas del Paraguay } \\
\text { Ruta de la Caña Paraguaya } \\
\text { Chipá Rapé } \\
\text { Circuito Gastronómico Asunción } \\
\text { Circuito Gastronómico Alto Paraná }\end{array}$ \\
\hline Perú & $\begin{array}{l}\text { Ministerio de Comercio Exterior y } \\
\text { Turismo }\end{array}$ & $\begin{array}{l}\text { Cocina de la costa norte } \\
\text { Cocina de la Amazonía } \\
\text { Cocina de Lima } \\
\text { Cocina de Arequipa } \\
\text { Cocina andina } \\
\text { Cocina Novoandina } \\
\text { El grano de Oro - La quinua } \\
\text { Pisco } \\
\text { El aguaymanto } \\
\text { El Camu Camu } \\
\text { Chirimoya } \\
\text { La Lúcuma } \\
\text { La Kiwicha } \\
\text { Perú, mucho gusto } \\
\text { Mistura } \\
\text { Semana del Chilcano } \\
\text { Ruta del Pisco }\end{array}$ \\
\hline Uruguay & Ministerio de Turismo del Uruguay & $\begin{array}{l}\text { Inmersos en la Ruta } 5 \text { Sentidos } \\
\text { Fiestas Tradicionales } \\
\text { Tomar un mate } \\
\text { Ir a comer un asado } \\
\text { Comer tortas fritas cuando llueve } \\
\text { Dieta uruguaya }\end{array}$ \\
\hline Venezuela & $\begin{array}{l}\text { Ministerio del Poder Popular para el } \\
\text { Turismo }\end{array}$ & Ruta del Café \\
\hline
\end{tabular}

Fuente: Elaboración propia a partir de los sitios web de los organismos nacionales de promoción turística de cada país.

En la tabla 1 anterior se observa la gran diversidad de recursos alimentarios, culinarios y gastronómicos que se utilizan para la promoción de los destinos. A partir del caso analizado de los países de América del Sur se puede confirmar que la gastronomía se incluye en la actualidad de forma generalizada en la promoción de los destinos turísticos.

Los datos demuestran que esto es especialmente relevante en casos como Argentina, Colombia, Ecuador o Perú. Esto no significa que los países que en la tabla anterior cuentan con pocos ejemplos tengan un menor interés en la gastronomía como factor de atracción turística. Esta afirmación requeriría un análisis más amplio de las ofertas turísticas nacionales y regionales, siendo esta una de las limitaciones de la presente investigación.

Sea como sea, todos los países analizados incluyen de una u otra forma el ingrediente gastronómico en su promoción turística. Los mensajes que se han podido obtener de los sitios web muestran los motivos porqué la gastronomía se utiliza de forma tan recurrente en el marketing de destinos. Por ejemplo, en el caso de Argentina, que se promociona como un país food friendly, se destaca que se trata de: 
"Un país donde los sabores hablan de la diversidad natural y cultural de los pueblos que lo integran. $Y$ donde se elaboran vinos que asombran al mundo. Variados, coloridos, irresistibles y con identidad propia, así son los sabores de Argentina" (INSTITUTO NACIONAL DE PROMOCIÓN TURÍSTICA DE ARGENTINA, 2017).

En la misma dirección se encuentran los mensajes promocionales de la gastronomía en Ecuador:

\begin{abstract}
"La gastronomía ecuatoriana se caracteriza por una riqueza de sabores por la agro-biodiversidad, multiculturalidad y herencia ancestral. Por eso es fácil encontrar diversidad de bebidas elaboradas con granos o hierbas, carnes, pescados y mariscos y platos que se sirven en determinadas épocas del año. De región a región hay diferentes preparaciones, técnicas de cocción e ingredientes que convierten la visita a cada lugar en una experiencia gastronómica" (MINISTERIO DE TURISMO DEL ECUADOR, 2017).
\end{abstract}

La gran diversidad gastronómica dentro de cada país es pues relevante, y hace que en algunos casos la oferta gastronómica se presente dividida por regiones, productos estrella o tradiciones culinarias. $\mathrm{A}$ la vez, estas tradiciones culinarias son también una amalgama de culturas, donde la inmigración ha jugado un papel fundamental. Esto también se observa a continuación para el caso peruano:

\begin{abstract}
"La cocina peruana, considerada como una de las más privilegiadas del mundo, heredó de la historia su ingenio, su mestizaje y su sabor. La fusión de su cocina se debe al intercambio cultural a través del tiempo, donde destacan la inmigración española, africana, china, japonesa e italiana. La variedad de sus platos nació a medida que el mestizaje crecía o mientras los migrantes llegaban al puerto de Callao" (MINISTERIO DE COMERCIO EXTERIOR Y TURISMO DEL PERÚ, 2017).
\end{abstract}

En la promoción del turismo gastronómico de Chile también se la da importancia a la mezcla, en la cocina chilena, de las tradiciones indígenas con las tendencias gastronómicas europeas. En la mayoría de los casos se destaca también la combinación de los distintos paisajes gastronómicos, vinculados al mar y a la tierra, y confiriendo un rol primordial al vino, como se veía anteriormente para el caso de Argentina. Sin dejar el caso chileno, su oferta gastronómica se diferencia en tres direcciones (MINISTERIO DE ECONOMÍA, FOMENTO Y TURISMO DE CHILE, 2017): la primera, los restaurantes, bares y cafés que ofrecen una gran variedad de gastronomías del mundo; la segunda, los platos de gastronomía típica con ingredientes como la quinoa, las preparaciones con maíz, los pescados y mariscos de la costa chilena o las carnes en zonas como la Patagonia; y la tercera, el enoturismo, los valles y las zonas productoras así como las fiestas de la vendimia que se celebran entre marzo y abril cada año.

El vino, junto con otros productos como el maíz, la mandioca 0 las legumbres son recurrentes en la mayoría de tradiciones culinarias de América del Sur. Esto se observa en la promoción de la gastronomía paraguaya, que al mismo tiempo trata de diferenciarse a partir de sus señas de identidad cultural y natural:

\begin{abstract}
"La gastronomía del Paraguay ha tenido en los últimos años un gran despegue manteniendo la base de la gastronomía tradicional con los productos del agro y las carnes, pero fusionándola con productos de la gastronomía internacional. La herencia de los recursos naturales de los Guaraníes y la amalgama con la cultura europea nos dan una gastronomía diferente al resto de América, en la que sobresalen las delicias como la chipa. La sopa paraguaya, el chipa guazú, el soyo, el borí borí, entre otros, además de una gran variedad de postres" (SECRETARÍA NACIONAL DE TURISMO DE PARAGUAY, 2017).
\end{abstract}

Las idiosincrasias culturales y naturales como razones de peso en el marketing gastronómico también son presentes en el caso de Colombia, ya que "la diversidad cultural y climática del país le da origen a un menú variopinto de platos típicos que se extiende de La Guajira al Amazonas y del Pacífico a Ios Llanos Orientales" (MINISTERIO DE COMERCIO, INDUSTRIA TURISMO DE COLOMBIA, 2017). AI mismo tiempo, la cultura cafetera también ocupa un lugar importante en la promoción de la identidad colombiana.

Otros países posicionan su gastronomía como una actividad dentro de la práctica turística a partir de iniciativas concretas como por ejemplo los productos típicos del Mercado Municipal de São Paulo (EMPRESA BRASILEÑA DE TURISMO, 2017), costumbres de gran tradición como el consumo del mate en Uruguay (MINISTERIO DE TURISMO DEL URUGUAY, 2017), la producción de café como un elemento de proyección internacional en Venezuela (MINISTERIO DEL PODER POPULAR PARA EL TURISMO, 2017) o la referencia a una plataforma que aglutina los negocios gastronómicos de Bolivia (EMPRESA ESTATAL BOLIVIANA DE TURISMO, 2017).

Todos estos ejemplos de productos, actividades culturales, experiencias y países son una muestra de la fuerte inclusión de la gastronomía en la promoción turística en la actualidad. La forma cómo se transmiten los mensajes evidencia a la vez los principales argumentos que se utilizan para dar visibilidad a la gastronomía a través de su inclusión en la práctica del turismo. Cabe recordar de nuevo que los ejemplos mencionados a lo largo de este artículo son solo una muestra de la gastronomía de los países 
de América del Sur. Un estudio más detallado de la oferta gastronómica debería incluir también los organismos de gestión y promoción turística a otros niveles territoriales como las regiones y las ciudades, así como las particularidades que pudieran derivarse del estudio de empresas turísticas, agencias de viajes o blogs y redes de turismo gastronómico, entre otros.

\section{CONCLUSIONES}

Este artículo estudia el fenómeno del marketing gastronómico a partir de la promoción que se hace de la gastronomía a nivel nacional. El contexto actual, donde la gastronomía ha adquirido en las últimas dos décadas una importancia sin precedentes, hace que los promotores del turismo incluyan siempre los recursos gastronómicos como uno de los principales factores de atracción.

No obstante, el componente gastronómico, vinculado a los productos, los restaurantes o los eventos, siempre ha sido parte importante del viaje turístico, y por lo tanto del gasto de los turistas. Aunque si se atiende a la reciente trayectoria de la literatura académica existente, la promoción de la gastronomía pueda considerarse hasta cierto punto innovadora, esto solo se puede justificar desde el punto de vista de las infinitas posibilidades que el fenómeno gastronómico ofrece.

Por muy básico que parezca, esto nos remite a la principal definición sobre turismo gastronómico y que continúa vigente hoy en día (HALL; SHARPLES, 2003). Esta definición incluye tanto el consumo directo de los productos como el indirecto, más relacionado por ejemplo con las visitas a los productores o los eventos, entre otros ejemplos de experiencias gastronómicas mencionados en la sección anterior.

La principal aportación que este artículo realiza en el contexto de la promoción de la gastronomía y del turismo gastronómico en los destinos es que, a partir del caso de estudio de los países de América del Sur, se puede observar que la gastronomía es un factor de atracción de los destinos que, en concreto, se manifiesta principalmente a través de los propios alimentos, las experiencias gastronómicas, y la oferta particular de cada región.

En primer lugar, pues, los productos son los que transmiten la autenticidad del lugar, de su naturaleza y su cultura, de sus gentes, y por lo tanto se erigen como marcadores y símbolos de la identidad territorial y el sentido de pertenencia al lugar. Esto les proporciona un significado clave para la atracción de turistas.

En segundo lugar, las experiencias turísticas basadas en la gastronomía son una forma de vertebrar la oferta - a través de los propios productos, o de los platos tradicionales, por ejemplo - que introducen a los turistas en el contexto local de una forma vivencial.

En este sentido, las experiencias les permiten participar en primera persona de las tradiciones locales y conocer la historia y las historias que se esconden detrás de ellas. En tercer lugar, las regiones como zonas geográficas son el tercer elemento a través del cual los destinos promocionan su gastronomía. A la vez, estas aúnan productos y experiencias, sumando esfuerzos como espacios territoriales para así obtener una mayor visibilidad y un impacto regional más amplio.

Los ejemplos observados demuestran que la gastronomía se incluye de forma generalizada en la promoción turística de los países, y por lo tanto confirman el papel clave que la gastronomía desempeña en la comercialización de los destinos turísticos (CHAVES; COSTA, 2014; DURAND; HEATH; ALBERTS, 2003; OKUMUS; OKUMUS; MCKERCHER, 2007). Esto se debe a que se trata de una forma de diferenciación, especialización y competitividad de los destinos, tal y como se veía al principio.

A la vez, la promoción de la gastronomía se integra en el contexto cultural, histórico y natural de los destinos, lo cual se observa a través de los festivales, rutas 0 circuitos gastronómicos. No obstante, la relación de los recursos gastronómicos que pueden mostrar la autenticidad e identidad de los países es mucho más amplia que la relacionada en este artículo y responde también a particularidades regionales que en algunos casos no se reflejan en este estudio.

Como se apuntaba con anterioridad, esto representa a la vez una limitación y una oportunidad de investigación futura. También el carácter descriptivo del presente artículo es una de sus limitaciones principales.

Entre las contribuciones teóricas y prácticas de la promoción de la gastronomía para la atracción de turistas que se derivan de este artículo, se ha podido observar cuáles son los elementos que sustentan la oferta turística gastronómica de los países de América del Sur. Esto permite conocer hacia dónde se orienta la gestión nacional de la oferta turística de los destinos y qué estrategias de comunicación y comercialización se utilizan a partir de las prácticas de turismo gastronómico, lo cual puede beneficiar ampliamente el desarrollo regional.

A la vez, es importante mencionar que por ejemplo Crockett y Wood (1999) afirman que para conseguir una marca estatal es necesario disponer de varias marcas secundarias, o regionales, que tienen también un gran interés en la construcción de la marca-país (LEE; ARCODIA, 2011). 
En el contexto del uso y evaluación de las estrategias de mercadotecnia relacionadas con el turismo gastronómico, los destinos deben tener en cuenta varios aspectos, de acuerdo con Du Rand, Heath y Alberts (2003, p.109): una mayor utilización de los alimentos locales frente a los de importación; el uso de la cocina y la gastronomía como un elemento clave en la imagen de marca del destino; así como la vertebración de la oferta entorno a rutas 0 establecimientos especializados que actúen como polos de promoción de las diferentes identidades culinarias. Todo ello representa una contribución del turismo gastronómico a la competitividad de los destinos y a su desarrollo sostenible (DU RAND; HEATH, 2006).

Investigaciones posteriores en el campo del marketing turístico en su enfoque gastronómico deberían analizar cómo se puede mejorar la promoción de la gastronomía. Especialmente, en un contexto donde la segmentación de los turistas gastronómicos se antoja ya imprescindible (KIVELA; CROTTS, 2005).

Así, de acuerdo con otros autores, es importante conocer cómo el comportamiento de compra de los turistas se ve influido por el consumo de alimentos locales a través del análisis de la repercusión que tienen las estrategias específicas de marketing (OKUMUS; OKUMUS; MCKERCHER, 2007).

Esto ayudaría a diseñar acciones más efectivas. Además, por un lado, es importante conocer el rol que determinados recursos juegan en la creación de la imagen de marca y en la satisfacción de los visitantes. Por el otro, resulta imprescindible analizar el grado de identificación de los actores locales con las señas de identidad gastronómicas que se utilizan en el marketing de los destinos turísticos.

A la vez, es necesario profundizar en si las representaciones que el marketing hace de la gastronomía local, implican una (in)correcta comprensión e interpretación del sentido de lugar. Por último, el comportamiento de los consumidores turísticos y el retorno económico de las acciones de marketing gastronómico es otro aspecto trascendental para calibrar el impacto de la gastronomía en el desarrollo de los destinos turísticos.

\section{REFERENCIAS}

BARROCO, C.; AUGUSTO, L. Turismo Gastronómico em Portugal: Formas de Comunicar os Produtos Endógenos da Região dão Lafões e Alto-Paiva. Anais Brasileiros de Estudos Turísticos - ABET, 6(2), 23-39, 2016

BERNO, T.; LAURIN, U.; MALTEZAKIS, G. The special role of agriculture in food tourism. En: E. Wolf (Ed.), Have fork will travel: Handbook for food tourism, 105-114. Seattle: World Food Travel Association, 2014.

BESSIĖRE, J. Local development and heritage: Traditional food and cuisine as tourist attractions in rural areas. Sociologia Ruralis, 38(1), 21-34, 1998.

BESSIĖRE, J. The role of rural gastronomy in tourism. En: L. Roberts y D. Hall (Eds.), Rural Tourism and Recreation: Principles to Practice, 115-118. Wallingford: CABI International, 2001.

BIGNÉ, E. Turismo y Marketing. Una revisión y perspectivas de futuro. Estudios Turísticos, 129, 105127, 1996.

BIGNÉ, E. Nuevas orientaciones del marketing turístico: de la imagen de destinos a la fidelización de los turistas. Papeles de Economía, 102, 221-235, 2005.

BIGNÉ, E. Las respuestas del turista ante la imagen del lugar de origen del producto. En: C. FLAVIÁN; C. FANDOS (Coords.), Turismo gastronómico: Estrategias de marketing y experiencias de éxito, 5168. Zaragoza: Prensas Universitarias de Zaragoza, 2011.

BIGNÉ, E.; FONT, X.; ANDREU, L. Marketing de destinos turísticos. Madrid: Esic Editorial, 2000.

BJÖRK, P.; KAUPPINEN-RÄISÄNEN, H. Local food: a source for destination attraction. International Journal of Contemporary Hospitality Management, 28(1), 177194, 2016.

BLASCO, A. Barcelona Atracción (1908-1936): Una revista de la Sociedad de Atracción de Forasteros de Barcelona. Papers de Turisme, 45, 79-80, 2009.

CABRERA, S. Marketing gastronómico. La experiencia de convertir el momento del consumo en un recuerdo memorable. Centro de Estudios en Diseño y Comunicación, 45, 165-174, 2013.

CALVO, D. Productos agroalimentarios de calidad, gastronomía y patrimonio cultural: activos para la promoción del territorio y de destinos turísticos. En: $\mathrm{C}$. FLAVIÁN; C. FANDOS (Coords.), Turismo gastronómico: Estrategias de marketing y experiencias de éxito, 93-116. Zaragoza: Prensas Universitarias de Zaragoza, 2011.

CHAVES PIMENTEL, M.P.; COSTA MACHADO, D.F. Entre a Fome de Distinção Social e os Prazeres da Gula: uma discussão sobre os significados culturais associados ao consumo no turismo gastronômico. Anais Brasileiros de Estudos Turísticos - ABET, 4(2), 19-31, 2014.

CHEN, Q.; HUANG, R. Understanding the importance of food tourism to Chongqing, China. Journal of Vacation Marketing, 22(1), 42-54, 2016.

CROCE, E.; PERRI, G. Food and Wine Tourism: Integrating Food, Travel and Territory. Oxfordshire: CAB International, 2011.

CROCKETT, S.R.; WOOD, L.J. Brand Western Australia: a totally integrated approach to destination branding. Journal of Vacation Marketing, 5, 276-289, 1999.

CROUCH, G.I.; RITCHIE, J.R.B. Tourism, competitiveness and social prosperity. Journal of Business Research, 44, 137-152, 1999. 
COHEN, E.; AVIELI, N. Food in Tourism: Attraction and Impediment. Annals of Tourism Research, 31(4), 755778, 2004.

DU RAND, G.E.; HEATH, E. Towards a Framework for Food Tourism as an Element of Destination Marketing. Current Issues in Tourism, 9(3), 206-234, 2006.

DU RAND, G.E.; HEATH, E.; ALBERTS, N. The Role of Local and Regional Food in Destination Marketing. Journal of Travel and Tourism Marketing, 14(3-4), 97112, 2003.

EMPRESA BRASILEÑA DE TURISMO, 2017. www.visitbrasil.com/es [Fecha de consulta: 10 de julio de 2017].

EMPRESA ESTATAL BOLIVIANA DE TURISMO, 2017. www.bolivia.travel [Fecha de consulta: 10 de julio de 2017].

EVERETT, S. Iconic cuisines, marketing and place promotion. En: D. TIMOTHY (Ed.), Heritage Cuisines: Traditions, identities and tourism, 119-131. New York: Routledge, 2016.

FERRERO, S. Comida sin par. Consumption of Mexican Food in Los Angeles: Foodscapes in a transnational consumer society. En: W. BELASCO; P. SERANTON (Eds.), Food Nations: Selling Taste in Consumer Societies, 194-219. New York: Routledge, 2002.

FRUSTIER, P. La communication touristique des territoires. Bresson (Isère): Groupe territorial, Voiron, 2009.

FUSTÉ FORNÉ, F. El turisme gastronòmic: autenticitat i desenvolupament local en zones rurals. Documents d'Anàlisi Geogràfica, 61(2), 289-304, 2015.

FUSTÉ FORNÉ, F. Tasting cheesescapes in Canterbury (New Zealand). New Zealand Geographer, 72, 41-50, 2016a.

FUSTÉ FORNÉ, F. Cheese Tourism: local produce with protected designation of origin in the region of Galicia, Spain. En: C.M. HALL; S. GÖSSLING, S. (Eds.), Food Tourism and Regional Development: Networks, products and trajectories, 242-252. Abingdon: Routledge, 2016b.

GYIMÓTHY, S.; MYKLETUN, R.J. Scary food: Commodifying culinary heritage as meal adventures in tourism. Journal of Vacation Marketing, 15(3), 259273, 2009.

HALL, C.M. Wine food and tourism marketing: Preface. Journal of Travel and Tourism Marketing, 14, 2003.

HALL, C.M. Heirloom products in heritage places: Farmers markets, local food, and food diversity. En: D. TIMOTHY (Ed.), Heritage Cuisines: Traditions, identities and tourism, 88-103. Abingdon: Routledge, 2016.

HALL, C.M.; SHARPLES, L. The consumption of experiences or the experience of consumption?: An introduction to the tourism of taste. En: C.M. HALL; L. SHARPLES; R. MITCHELL; N. MACIONIS; B. CAMBOURNE (Eds.), Food tourism around the world: Development, management and markets, 1-24. Oxford: Elsevier, 2003.

HALL, C.M.; SHARPLES, L.; MITCHELL, R.; MACIONIS, N.; CAMBOURNE, B. Food tourism around the world:
Development, management, and markets. Oxford: Elsevier, 2003.

HENDERSON, J.C. Food tourism reviewed. Bristish Food Journal, 111(4), 317-326, 2009.

HERNÁNDEZ, J. La imagen de Andalucía en el turismo. Sevilla: Fundación Centro de Estudios Andaluces, Junta de Andalucía, 2008.

HILLEL, D., BELHASSEN, Y.; SHANI, A. What makes a gastronomic destination attractive? Evidence from the Israeli Negev. Tourism Management, 36, 200-209, 2013.

HJALAGER, A.M.; CORIGLIANO, M.A. Food for tourists: determinants of an image. International Journal of Tourism Research, 2(4), 281-293, 2000.

HJALAGER, A.M.; RICHARDS, G. Tourism and gastronomy. New York: Routledge, 2002.

INSTITUTO NACIONAL DE PROMOCIÓN TURISTICA DE ARGENTINA, 2017. www.argentina.travel/es [Fecha de consulta: 21 de junio de 2017].

KIM, Y.G.; EVES, A. Construction and validation of a scale to measure tourist motivation to consume local food. Tourism Management, 33(6), 1458-1467, 2012.

KIVELA, J.; CROTTS, J.C. Gastronomy Tourism: A Meaningful Travel Market Segment. Journal of Culinary Science and Technology, 4(2-3), 39-55, 2005.

KIVELA, J.; CROTTS, J.C. Tourism and gastronomy: gastronomy's influence on how tourists experience a destination. Journal of Hospitality and Tourism Research, 30(3), 354-377, 2006.

KOTLER, P.; GARCIAA DE MADARIAGA, J.; FLORES, J.; BOWEN, J.T.; MAKENS, J.C. Marketing turístico. Madrid: Pearson Educación, 2011.

LEE, A.H.J.; WALL, G.; KOVACS, J.F. Creative food clusters and rural development through place branding: Culinary tourism initiatives in Stratford and Muskoka, Ontario, Canada. Journal of Rural Studies, 39, 133-144, 2015.

LEE, I.; ARCODIA, C. The Role of Regional Food Festivals for Destination Branding. International Journal of Tourism Research, 13, 355-367, 2011.

LONG, L. Culinary tourism and the emergence of an Appalachian cuisine: Exploring the "foodscape" of Asheville. North Carolina Folklore Journal, 57(1), 419, 2010.

MINISTERIO DE COMERCIO, INDUSTRIA Y TURISMO DE COLOMBIA, 2017. www.colombia.travel/es [Fecha de consulta: 21 de junio de 2017].

MINISTERIO DE COMERCIO EXTERIOR Y TURISMO DEL PERÚ, 2017. www.peru.travel/es-pe [Fecha de consulta: 22 de junio de 2017].

MINISTERIO DE ECONOMÍA, FOMENTO Y TURISMO DE CHILE, 2017. www.chile.travel [Fecha de consulta: 22 de junio de 2017].

MINISTERIO DE TURISMO DEL ECUADOR, 2017. www.ecuador.travel.es [Fecha de consulta: 26 de junio de 2017].

MINISTERIO DE TURISMO DEL URUGUAY, 2017. www.turismo.gub.uy [Fecha de consulta: 10 de julio de 2017]. 
MINISTERIO DEL PODER POPULAR PARA EL TURISMO, 2017. www.mintur.gob.ve [Fecha de consulta: 10 de julio de 2017].

OKUMUS, B.; OKUMUS, F.; MCKERCHER, B. Incorporating local and international cuisines in the marketing of tourism destinations: The cases of Hong Kong and Turkey. Tourism Management, 28, 253261, 2007.

ORGANIZACIÓN MUNDIAL DEL TURISMO. Global Report on Food Tourism. Madrid: UNWTO, 2012.

RICHARDS, G. Gastronomy: An essential ingredient in tourism production and consumption? En: A.M. HJALAGER; G. RICHARDS (Eds.), Tourism and Gastronomy, 3-20. London: Routledge, 2002,

RITCHIE, J.R.B.; CROUCH, G.I. The competitive destination: A sustainability perspective. Tourism Management, 21, 1-7, 2000.

ROYO, M. El producto agroalimentario como atributo de importancia en la formación de la imagen del destino turístico. En: C. FLAVIÁN; C. FANDOS (Coords.), Turismo gastronómico: Estrategias de marketing y experiencias de éxito, 69-92 Zaragoza: Prensas Universitarias de Zaragoza, 2011.

SECRETARÍA NACIONAL DE TURISMO DE PARAGUAY, 2017. www.visitparaguay.travel [Fecha de consulta: 12 de julio de 2017].

SIDALI, K.L.; KASTENHOLZ, E.; BIANCHI, R. Food tourism, niche markets and products in rural tourism: combining the intimacy model and the experience economy as a rural development strategy. Journal of Sustainable Tourism, 23(8-9), 1179-1197, 2015.

SIMS, R. Food, place and authenticity: Local food and the sustainable tourism experience. Journal of Sustainable Tourism, 17(3), 321-336, 2009.

TRESSERRAS, J.J.; MEDINA, F.X. Patrimonio gastronómico y turismo cultural en el Mediterráneo. Barcelona: Ibertur, 2008.

WEILL, A. Pròleg a Imatge i destí. Cartells turístics de les comarques gironines. Girona: Museu d'Art de Girona, 2003.

YIN, R.K. Case study research: Design and methods. Thousand Oaks, CA: Sage Publications, 2014.

Processo Editorial / Editorial Process

Editor Chefe/Editor-in-chief: PhD Thiago D. Pimentel (UFJF).

Recebido em 25 de julho de 2017; aceito em 10 de dezembro de 2017; publicado online em 28 de Fevereiro de 2018.

Received on July 25, 2017; accepted on December 10, 2017, published online on February 28, 2017.

Artigo original / Original article. Seção revisada por pares / Double bind review section. 